\title{
Non-Locality: Possibility of Corpuscular-Wave Duality Explanation
}

\author{
Alexander Boichenko \\ Institute of Fundamental Problems in Theoretical Physics and Mathematics, Moscow, Russia \\ Email: boichenko.ifptpm@gmail.com
}

\begin{abstract}
The question of the nature of particles again and again arises as ideas about the world around us develop. The mysterious behavior of particles is reflected in the concept of corpuscularwave duality, which first appears as a result of examining experiments with light. The question of what constitutes light is one of the most ancient questions. In the work at the modern level of physical representations, an answer is offered to the question of what a particle is.
\end{abstract}

Keywords: Non-locality, dimension of space, corpuscular-wave duality, string theory, nature of particle.

\section{Introduction}

The current state of science is closely related to the physical properties of the environment in which the birth, formation and development of mankind took place. At an early stage of development, the ideas of primary matter, the structure of matter, symmetry are brought to the forefront of research [1].

The transition to a qualitatively different level, to an accurate description of physical phenomena, according to [2], was promoted by the development of astronomy. As a result of the development of astronomy, i.e. as a result of observing the movement of the planets, the concepts of central forces responsible for their movement appear. Respectively, to simplify the description, the interacting bodies are replaced by points, in other words, corpuscles. The shape of shadows resulting from the interaction of sunlight with various barriers leads to the idea of light as a stream of some particles - corpuscles. The concept of particle-corpuscle firmly enters the apparatus of scientific research. Thanks to the introduction of the concept of particle-corpuscle, it becomes possible to quantitatively describe the observed phenomena, as well as to formulate the laws of the world order and their further verification in observations or experiment. The formation and development of physics begins, because, as N. N. Bogolyubov considered, physics is what is located by measure, number and weight (in such way the "Book of Wisdom of Solomon" characterizes the universe created by God).

However, the intuitively clear meaning attributed to the concept of a particle gradually eroded as science developed. The paper presents an attempt to answer the question of what, at the current level of research, is to be understood as a particle.

\section{Local Description}

\subsection{Corpuscles or Waves?}

Corpuscles. It is convenient to observe the behavior of particles-corpuscles. On the basis of the analysis of their motion, one can already make detailed assumptions about the nature of their behavior. Naturally, a detailed start of physical research was begun with astronomy. First, in apparent disorder, harmony was found, which then led to dynamic equations describing the behavior of particles.

The new form, as a description of phenomena through dynamic laws acting on particles-corpuscles, with its further development led to the emergence of a local approach as the basis for describing the world around us. The behavior of such particles of the system under consideration is analyzed on the basis of a variational principle, whose action

$$
S=\int d t L
$$


where $L$ is the Lagrangian of the system, is based on the introduction of a local Lagrange function. Respectively, the behavior of light as a flux of corpuscles is analysed in geometrical optics based on the Fermat principle.

Further significant advances in the physics associated precisely with the use of Lagrangian functions with local density. The Lagrange function (Lagrangian) or its density is determined on spatial structures, the simplest of which is the point of space. Such Lagrange functions are called local. In this case, the Lagrangian

$$
L=L\left(t, x^{\mu}, \dot{x}^{\mu}\right), \mu=1, \ldots, N-1
$$

depends on the coordinates and velocities of point objects in the enclosing space of dimension $N$. In the local approach, the overwhelming number of results known to us is obtained.

However, as it turned out, the success of a dynamic description of the surrounding world based on a local dynamic approach to the motion of particles put forward by Newton has its limitations. Indeed, it turned out that there are a large number of phenomena, the explanation of which is possible only from the wave and is impossible from corpuscular positions. An example of such phenomena can be called the phenomena of interference and diffraction, known since the XVII century.

Waves. At the end of the XVII century, C. Huygens proposed the wave theory of light and gave remarkable explanations for the phenomena of reflection, refraction and double refraction, based on the concepts of waves and on the principle that now bears his name.

At the beginning of the XIX century, the works of T. Young again attracted attention to the phenomena of interference. A little later, E. Malus discovers the phenomenon of light polarization.

O. Fresnel shows that the phenomena of interference and diffraction are completely explained by the wave theory of light, while the corpuscular theory was unable to do so. Complementing Huygens' work, he shows that the theory of waves explains the rectilinear propagation of light rays in homogeneous media. Based on the hypothesis of transverse light waves, he creates a complete theory of polarization and double refraction. J. Maxwell gives the Fresnel waves an electromagnetic interpretation. Wave theory for some time held a strong position.

\subsection{Corpuscular-Wave Duality}

However, as it turned out, the corpuscular theory cannot be completely discounted. This is clearly understood I. Newton. After the discovery of the phenomenon of interference, which has since been called "Newton's rings", he made an interesting attempt to synthesize wave and corpuscle points of view in his "theory of attacks", which unfortunately remained in the germinal state and quickly forgotten.

In the local approach L. de Broglie from the analogy of the description of particles in classical mechanics and the wave motion of light, concludes that massive as well as massless particles, should exhibit wave properties and obtain a relation connecting the momentum of a particle with its wavelength [3]. This analogy is the heuristic factor of such a substantiation of the wave properties of particles. There is no derivation as such in the local approach.

The question of the nature of not only light, but also of particles, is already emerging at a higher level. What is decisive? Does wave behavior somehow determine corpuscular or, on the contrary, does corpuscular determine wave behavior of particles?

Corpuscle. A. Einstein consistently defended the point of view of the electromagnetic field as a gas of special elementary particles-corpuscles (photons). He assigned the role of "ghosts" to electromagnetic fields, which do not possess either energy or momentum, but only direct to the photons possessing these magnitudes.

The photo effect is explained by Einstein by light quanta (photons) in a purely corpuscular manner. In the light with wave frequency $v$ energy is concentrated in "granules" with $\hbar v$ portions. Einstein understood that his theory is not strictly corpuscular, because he uses the concept of frequency, which has a wave origin. As well as when considering problems of blackbody radiation, fluctuations of the black radiation energy density are divided by him into two members, one of which reflects the corpuscular structure, and the other - the wave nature. Nevertheless, considering the thermal equilibrium of gas molecules and black radiation in a certain volume with a uniform temperature, he showed that photons with energy $\hbar v$ must have momentum $\hbar v / c$ and that an atom emitting a photon experiences a recoil effect. 
In addition, in his opinion it is very problematic to believe that with the help of a regular wave one can get a complete picture of physical reality, as it does not contain any element that allows one to isolate a particle in it. In his ideas about photons, he also relied on the theory of relativity and relativistic concepts he was actively promoting. Light emission should be accompanied by some change in the source mass, therefore the light is no longer represented as oscillations of a certain medium, but as borrowed from the substance of the radiating body, which brings us closer to the corpuscular theory of light. Einstein's argument is also based on the radiation detection process. During registration, all photon energy is detected at one point, and not smeared over the entire space.

At the early stage of the development of quantum mechanics, it was possible to guarantee the validity of Planck's formula by assuming that the processes of emission and absorption of an electromagnetic field are probabilistic in nature $[4,5]$. As a result, physical processes acquired a statistical nature, and at the level of elementary processes, classical conservation laws turned out to be not obligatory. Even such a conservative in the matter of energy conservation as M. Planck did not object to such an approach.

After M. Born clarified the meaning of the wave function, W. Heisenberg noted that quantum mechanics in its form at that time already prescribed a physical interpretation for some of its values, for example, for average temporal values of energy, electric moment, momentum, etc. and, apparently, there was no longer any freedom of physical interpretations. Although the physical interpretation of the probabilistic laws of quantum mechanics was strictly fixed, the description nevertheless remained statistical.

However, in 1923, A. Compton observed a change in the frequency of electromagnetic radiation when scattered by electrons. This phenomenon was almost immediately explained by him and P. Debye as electron scattering on a particle (photon), which was confirmed in the experiment of B. Bothe and G. Geiger (1925). This circumstance is considered to be direct evidence that the photon is a corpuscle and evidence of the conservation of energy and momentum in elementary acts.

Thus, the main emphasis, it would seem, should focus on the corpuscular structure of the particles.

Wave. The development of the consideration of de Broglie, which led to the understanding that each particle should correspond to a well-defined wavelength, was a dual solution theory: any continuous solution $\Psi$ of wave mechanics is in some way duplicated by a singular solution $v$ containing in general a moving singularity (i.e. a corpuscle, and having the same phase as the solution $\Psi$ ). In this approach, both solutions $v$ and $\Psi$ would therefore have the shape of a wave, the phase of which would be the same function of coordinates and time, and the amplitude of each wave would be completely different, because the amplitude $v$ would contain a singularity, and the amplitude $\Psi$ would be continuous. The movement of the singularity would be affected by all the obstacles that would affect the propagation of the wave formation with which it is associated, and thus would explain the existence of interference and diffraction.

Since it was assumed that wave $\Psi$ has the same phase as wave $v$, and that the particle singularity moves always, following the phase gradient, the possible trajectories of the particle coincide with the curves orthogonal to the surfaces of the same phase $\Psi$. De Broglie noted [6] that this forced to consider the probabilities of finding a particle at some point equal to the square of the amplitude, i.e. wave intensity $\Psi$. So, this was precisely the first essential characteristic that should have been attributed to wave $\Psi$ : the square of its amplitude $|\Psi|^{2}$ at a certain point should have given the probability density of the presence of a particle associated with it at that point. This principle, adopted from the very beginning by wave mechanics and necessary for building a theory of electron diffraction, was, however, only a direct transfer of what was long accepted in optics.

If we accept the photon idea, then in this case it can only mean one thing, as it was very well seen, starting from its first works in 1905, Einstein: the probability that a certain photon was present at some point in space is proportional to the square of the amplitude of the light wave, which is associated with it $[6]$.

E. Schrödinger consistently sought to identify a particle with a small wave packet, but this interpretation ran into a serious obstacle, since the wave packet always in the linear description has a tendency to quickly and continuously spread in space and therefore cannot represent a certain particle capable of long and stable existence.

The hydrodynamic pattern of propagation of the E. Madelung wave consists in examining some fictitious fluid, the density of which at each point in space at each moment in time would be equal to the intensity of the continuous wave and which would flow, remaining as the wave propagated along the 
"streamlines", which are easy to determine analytically. However, all the streamlines in this approach are put to equal conditions and do not allow to represent any of them as a particle trajectory.

The beginning of Born's research on the physical interpretation of the wave function was associated with the desire at the new level of rigor associated with the recently formed quantum-mechanical approach to clarify the meaning of Einstein's "ghost fields". As a result of this consideration, the motion of a particle was represented as a motion in terms of a probability distribution, but this probability itself changes in a causal manner over time in accordance with the Schrödinger equation.

So, if we briefly summarize the wave interpretation of the particle behavior, we see that the main focus of the research was on controlling, through one or another mechanism, the behavior of the propagating corpuscle by the wave structure associated with it. Consequently, this would seem to indicate the primacy of the wave structure of the particles.

Principle of complementarity. From the above consideration, it would seem, it can be concluded that the use of one or another mathematical apparatus can lead in each particular case to the necessary conclusions. However, according to N. Bohr, mathematics is not able to prove any physical truth, it can provide only a certain formalism, expressing certain relationships between physical quantities [7]. The way out was found in the principle of complementarity of Bohr, which he announced in September (the international congress dedicated to the memory of A. Volta) and October (V Solvay congress) 1927.

According to this principle, the refinement of quantum effects requires a change in the experimental setup and entails new opportunities for fundamentally uncontrollable effects between objects and measuring instruments. So data obtained under different conditions can not be covered by a single scheme. These data should be considered as complementary in the sense that only a combination of different phenomena can give a more complete picture of the properties of the object [7].

In other words, the corpuscle and the wave are "additional aspects of reality" that complement each other, excluding each other, and each of these two aspects is manifested in the experiment only to the detriment of the other [8].

Heisenberg joined the principle of complementarity of Bohr in 1929 [9]. In his work, he notes that the uncertainty relations express only the limits of applicability of concepts about a particle, but these relations are not enough for a complete interpretation of the formalism. To determine precisely the limits of applicability of classical concepts, it is necessary and sufficient, as Bohr has shown, the simultaneous use of corpuscular and wave representations.

What is a particle in terms of corpuscular-wave duality and the complementarity principle on the example of a neutron Heisenberg considers in [1]. For example, he notes, we very simply and easily use the word "neutron". But we are not able to give any definite image of a neutron and cannot say that, in fact, we understand this word. We use various images and present the neutron as a particle, then as a wave or wave packet. But we know that none of these descriptions is accurate. Obviously, the neutron has no color, smell, taste ... Ordinary ideas of geometry and kinematics about a particle, such as shape or movement in space, cannot be applied to elementary particles in a consistent way. If we want to give an exact description of an elementary particle (here we emphasize the word "exact"), then the only thing that can be used as this description is a probability function. Hence it is concluded that, in general, if we are talking about a "property," then the "to be" property does not fit without limitation to an elementary particle. There is only a tendency, the opportunity to be.

Residual questions. So, it would seem, in accordance with the above statement of Heisenberg, the answer to the question about the nature of corpuscular-wave duality should sound like this: the object manifests itself as a corpuscle or wave depending on the conditions in which it is set, i.e. depending on the conditions of the experiment. However, the validity of such a conclusion also seems to be questionable.

Note that in the local description we can only describe the wave properties, but not explain them [10]. In addition, the typical corpuscular behavior of particles also remains questionable. For example, in experiments demonstrating a photoelectric effect, light, as noted in many early studies, definitely manifests itself as corpuscles, but Einstein's explanation of the photoelectric effect phenomenon from the point of view of the corpuscular theory of photons is not the only possible one. The [10] notes that Planck also had suspicions about this and he was much more cautious in his statements, despite the fact that Einstein convinces him of his concept of light. Planck's dazzling success in explaining black body law was based on the assumption that radiant energy can be emitted or absorbed only in finite portions, does it imply a hidden recognition that in the light wave energy is not distributed in a continuous 
manner but concentrated in the form of granules, particles of light? But Planck began to doubt the validity of such a revolutionary derivation of his own ideas and, fearing to question the wave structure of light, tried to avoid this by using rather artificial assumptions [8]. Returning to the photo effect, we note that all the features of the photoelectric effect experiments can also be explained on the basis of the wave nature of light [10-13].

In most cases, both wave and corpuscular approaches are quite acceptable for describing particles, in particular, experimental data on the radiation of both laser and lamp sources are reproduced theoretically quite well [14-26]. However, this does not remove the question of the nature of corpuscularwave duality.

\section{$2.3 \quad$ Field Paradigm}

It was noted above that Einstein consistently defended the corpuscular view of the nature of light. On the other hand, he also consistently developed the theory of relativity, which is based on the field description. At the end of his life, he comes to the idea that the combination of the ideas of a continuous field with the idea of discrete material points in space seems to be contradictory. A consistent theory requires that all the elements that appear in it are continuous not only in time, but also in space, and in all points of space. A particle should not be some element imposed from the outside; rather, it should belong to the field structure itself and constitute a kind of local anomaly in it.

He gave a similar type of fields the expressive name "field with humps" [8]. The matter that acts on our senses, in fact, from his point of view, is nothing more than some large concentration of energy in a very small volume of space. A thrown stone, in this sense, is a field that changes and in which states with the greatest intensity of the field move in space with the speed of the stone.

Thus, a consistent field description of the particles would be natural. If a certain field theory can be completed with a certain representation of particles free from singularities, then the behavior of these particles will be determined only by differential equations of the field. This view, in particular, was based on its result, obtained jointly with J. Grommer in 1927 (also obtained a year earlier by G. Darmois) when considering the Hilbert-Einstein equations without the right part, that if there is some very small area, where the field takes on very large values, the movement in space of this small area over time must of necessity be represented by some world line, which is a geodesic line of space-time.

The field point of view on the nature of the particles was considered earlier as well. At the end of the XIX - beginning of the XX century, a field view of the electromagnetic nature of particles and mass was advanced in the works of J. Thomson, O. Heaviside, M. Abraham, H. Lorentz, and others. Now this possibility is also being considered (see, for example, [27, 28]). To overcome the characteristic difficulties associated with the divergence of field structures, in particular, the self-interaction of particles in such consideration, new approaches are being developed, for example, the description of fields on lattices [29], but the field approach of describing particles is still far from completion.

\subsection{Last Attempt to Clarify Wave-Particle Duality in a Local Approach}

De-Broglie associated the hope of clarifying the essence of corpuscular-wave duality with the further development of his theory of dual solutions. Having received an invitation to the V Solvay Congress, he presents a simplified version of the theory, known as the wave-pilot theory, for his report. After critical objections, de Broglie leaves the work in this direction for a long time and joins the Copenhagen school of interpreting quantum theory.

De Broglie's return to the theory of double solutions occurs after the publication D. Bohm's works in the 1950s. Bohm's article (1951) does not contain anything essentially new, because it only turns again to the pilot-wave theory, which was presented by de Broglie at the Solvay Congress, to a theory that introduces only a probability wave $\Psi$ and does not introduce a wave $v$ with a singularity used by the dual solution hypothesis. Bohm made a number of interesting observations and, in particular, carried out an analysis of the measurement processes, considering them from the point of view of the pilot wave, and which seems to have refuted the objections put forward by W. Pauli against the ideas of de Broglie in 1927 [6]. In fact, Bohm built his version of quantum mechanics, which included both non-locality and hidden parameters. In the Bohm approach, particles have well-defined characteristics (both position and velocity), although we cannot measure them simultaneously. However, this is achieved by introducing a 
more enhanced element of non-locality, which manifests itself in the fact that a particle is affected by forces that instantaneously change from conditions in remote locations [30].

De Broglie is also concerned about the philosophical component of his theory. To the question why change the modern interpretation (i.e., the principle of complementarity), if it is sufficient to explain all the observed phenomena, why introduce all these useless complications of the theory of dual solutions, solutions with singularities, etc., thereby risking getting lost in labyrinths? He replies that returning to clear Cartesian ideas, observing the validity of the figurative description within the framework of space and time, would definitely satisfy many minds and would avoid some strange conclusions arising from the modern interpretation based on a continuous function (wave function $\Psi$ ), the statistical nature of which is a kind of "subjectivism", akin to idealism in the philosophical sense, i.e. to the denial of the existence of a physical reality independent of the observer [6].

Jean-Pierre Vigier of the Henri Poincaré Institute, in the continuation of Bohm's attempt, expressed a very interesting idea about the connection between the theory of dual solutions and the theorem proved by Einstein in joint work with Grommer in 1927. If we consider the elementary particles of matter as singularities in a gravitational field, it was proved proceeding solely from the equations of the gravitational field that the motion of singularities occurs along the geodesic lines of space-time, without introducing this result as an independent postulate. This proof is in many respects analogous to that of de Broglie (also 1927), in which it was shown that the velocity of the particle should be directed along the phase gradient of wave $\mathrm{v}$, the singularity of which it represents.

In addition, analyzing the J. von Neumann theorem on the impossibility of the existence of hidden parameters, de Broglie notes a gap in its proof [6], which allows him to return to the theory of dual solution with new hopes, among which, in particular, the possibilities provided by introduction to the consideration of nonlinearity.

However, hopes were not justified. The problem of the impossibility of the presence in the quantum theory of hidden parameters is closely related to the problem of the non-locality of physical interactions in the microworld, which we consider in more detail below.

\subsection{Local Description. Results}

Thanks to the local description, we have achieved a significant advance in understanding the phenomena of the microworld. But does this support the local nature of interactions in nature? Apparently not. What is the evidence of the non-locality of interactions?

First, the very construction of the apparatus of quantum mechanics. The developed apparatus of quantum mechanics deals with wave functions. But the approach based on the use of wave functions is related to the probability description. We can only predict the probabilities of the processes that are taking place. This in itself already suggests that since we can not talk about exact localization, for example, the position and momentum of a particle, this circumstance may be due precisely to its nonlocality or its nonlocal interaction with the environment.

The non-locality of our world, in particular, is revealed, in addition to the already mentioned probability description, the corpuscular-wave duality and the Heisenberg uncertainty relations, also in the non-commutativity of quantum-mechanical operators, the necessity of taking into account all possible positions of the system (an alternative approach based on path integrals [31]), EPR-paradox [32, 33], Bell's inequalities [34] etc. [35].

The corpuscular-wave dualism reflects the fact that the system participates in all its possible realizations. This is one of the brightest manifestations of non-locality. For example, particles in interference experiments feel at once both (or all, if there are more than two) slits. An attempt to describe these experiments according to the local nature of particles does not lead to an explanation of the observed effects. Conducting ingenious experiments that seemingly should "force" photons or particles to behave locally - experiments on the interference of single particles, experiments on anticoincidence, experiments with deferred choice, quantum eraser, quantum eraser with deferred choice $[10,36]$, etc., each time lead to the opposite situation. Particles can not be deceived. They feel all their possible positions and participate in them and do not succumb to the provocation of these ingenious experiments [10].

In the previous paragraph, de Broglie's attempts to circumvent the absence of hidden parameters in quantum systems were mentioned. This would solve, as de Broglie believed, the problem of corpuscular- 
wave duality in a local approach in his theory of dual solution. The problem of the presence of hidden parameters in the system re-emerges in connection with the Bell inequalities. It turns out that this problem has been known in mathematics for a very long time.

The problem of the possibility of simultaneous realization of $\mathrm{n}$ random variables with given correlations among themselves, when these quantities are independent of each other, but depend on some other random variables, arises within the framework of the classical probability theory long before the appearance of Bell inequalities (1964). The necessary conditions for such an implementation were originally obtained by $\mathrm{J}$. Boole in the form of inequalities about 100 years before the introduction of Bell's inequalities, and the general solution for a system of $n$ random variables was obtained by $\mathrm{N}$. N. Vorobyov in 1962 [37].

In the case of two particles, Bell's inequalities, which connect three random variables that can take values \pm 1 , independent of each other, and depending on some random variable $\lambda$, introduced to analyze the Einstein-Podolsky-Rosen (EPR) mental experiment [32, 33]. Quantum mechanics presupposes a probabilistic description (see above), so if Bell's inequalities are satisfied for non-commuting observables, then this leaves hope for the presence of hidden parameters $\lambda$. In other words, quantum probabilities and uncertainties could be reduced in this case to the classical theory of probability, and the state of the system, thus, would be set not by a wave function, but by a set of hidden parameters.

However, consideration of Bell's inequalities as applied to the wave function of two particles with zero total moment (EPR state) shows that by choosing the directions of the axes on which the projections of angular moments or particle spins are measured, the Bell inequalities can be broken [38-40] for the correlators of the projections on the axis data $[10,37]$. This violation is known as the EPR paradox, however, this behavior of quantum correlated systems is paradoxical only from the classical local point of view. If we assume the presence of non-locality, then we have no paradox.

The generalization of Bell's inequalities in the case of three particles is carried out by D. Greenberger, M. Horn, A. Zeilinger (1989), starting from which a new stage in the study of non-locality in manyparticle systems opens [10].

So, the world surrounding us is non-local. It is better to describe a nonlocal nature in the corresponding nonlocal approach. Although, until now, we were quite satisfied the local approach in nonlocal phenomena understanding, but it is not entirely natural to investigate the nonlocal nature in the local description.

\section{Nonlocal Description}

\subsection{Features and Limitations}

The main result of the development of quantum mechanics was the understanding of the non-locality of our world. Local description was very good to understand the classical behavior of the objects surrounding us. Of course, with this local description frame of classical physics had to start studying the phenomena of the quantum world. It turned out that the apparatus of the local description is also suitable for describing nonlocal phenomena. Thus, for example, the theoretical value of the anomalous magnetic moment of an electron in relativistic quantum electrodynamics coincides with the experimental value with an accuracy of up to 11 significant digits (relative accuracy $210^{-10}$ ) [41-44].

However, with the development of the quantum theory, some illogicality of such a description has become more and more apparent. We use a local approach, but in doing so, you come to the fact that it is impossible exact determination of the positions and momenta of particles at the same time (the Heisenberg uncertainty relations). We can describe, if necessary, the corpuscular nature of particles, or, if necessary, its wave nature, but you can not in this approach say why the corpuscles suddenly begin to exhibit wave properties [10], etc. In addition, if we can guarantee the local behavior of particles in interference experiments, for example, if we can ensure in one way or another the passage of a particle only through one slit, then the wave manifestations disappear.

Therefore, we should initially develop non-local schemes.

String theory. As noted above, local Lagrange functions are defined on points. The object following on complexity after a point is the one-dimensional structure - the line. Using theories with local Lagrange functions greatly simplifies the analysis, but it does not follow from anywhere that it is the 
only possible physical theories. Lines on which Lagrange's function is defined, are called as strings, respectively, we deal with string theories [45-53]. The main emphasis of research with nonlocal Lagrangians is still focused on the consideration of strings. But we should remember that the onedimensional objects just a special case of non-local objects. In such approach it is necessary to analyze also objects of higher dimension - membranes $(n \geq 2)$ [54]. In the non-local case we have

$$
L=\int d q^{n} \tilde{L}\left(t, X^{\mu}\left(t, q^{\nu}\right), \frac{\partial X^{\mu}\left(t, q^{\nu}\right)}{\partial t}, \frac{\partial X^{\mu}\left(t, q^{\nu}\right)}{\partial q^{\lambda}}\right)
$$

where $\tilde{L}$ is the Lagrangian density, the functions $X^{\mu}\left(t, q^{\nu}\right)$ are the functions of mapping extended objects ( $n=1$ for a string, $n \geq 2$ for membranes) into enclosing space. The integration is performed over the volume of an extended object (with $n=1$ along the string, with $n=2$ along the surface of the object), described by the parameters $q^{v}(v=1, \ldots, n)$. String theory can really be productive if the contribution of membrane theories in the description of physical processes will be small. But the development of membrane theories practically not moving and this is due to their significant nonlinearity [47].

Basically, optimism when considering string theory is related to the fact that this approach can lead to quantization of gravity. Note that while all the advantages of string theory remain without experimental confirmation. Nevertheless, this approach is undoubtedly also necessary to develop, in order to understand what opportunities in the description of the real world it can provide.

The beginning of the development of the string approach to the description of physical phenomena belongs, apparently, to the end of the 60s of the twentieth century. In his lectures (Sicily, 1967), M. Gell-mann dwells on the specific features of the scattering amplitudes of protons and neutrons [55]. G. Veneziano became interested in these features, who a year later presented the function "magically" describing these features [56]. After another two years, it becomes clear that such properties of the Veneziano function are obtained from a theory in which protons and neutrons are small oscillating strings, and not point objects [57-59].

Note that the string approach has two aspects. On the one hand, this is an approach to the description of exclusively non-local phenomena. It is this aspect that appears in the above-mentioned works [57-59]. The considered strings were of a bosonic nature, many physicists believed that the Veneziano model lacked quarks at the ends of the string. Only later it was shown that the introduction of fermions and extension of a model, including both bosons and fermions [60-62], is possible.

On the other hand, its appearance is quite natural in hadron physics, as a consequence of the development of quantum chromodynamics. Hadrons are interpreted as bound states of spinor fields (quarks) with non-Abelian gauge fields of vector bosons (gluons). Due to the quark confinement hypothesis, it is highly likely that such configurations of gluon fields are energetically more favorable when these fields do not fill the whole space, but concentrate along the lines connecting the quarks, which naturally leads to the concept of strings.

The emergence of a model of relativistic strings also contributed the development of other areas of theoretical physics: electrodynamics with Dirac magnetic charges, nonlinear field models of the BornInfeld type, dual-resonant hadron models, bag models, etc. [47].

Dimension of space. The non-locality of the phenomena of the microworld must have very nontrivial consequences. When describing nonlocal objects in string theory, we come across fundamental aspects of this theory. The point is that string theories can not exist in a space of arbitrary dimension. The dimension of the space depends on the sector of interactions considered in this theory. For example, in the bosonic sector of theory this dimension must be 26, in the fermionic supersymmetric sector of theory it must be 11 [45-53] (the dimensionality of our real space can take greater values [63, 64]). Thus, the non-locality of the phenomena of the microworld indicates to us the fact that we are in a space of a certain dimension. In the local description, we also come to multidimensional spaces [63, 64], but in this case the transition to multidimensional spaces can be interpreted as the possibility of a more convenient description of the processes under consideration.

Cosmological constant. So, the problem of non-locality in quantum mechanics led to a consideration of a nonlocal description of micro phenomena. This consideration, in particular, led to the construction of nonlocal Lagrangians, or in other words to the construction of a string theory as the simplest variant of non-locality. In this theory, space can not have an arbitrary dimension - the dimension is determined by commutation relations for the generators of Lorentz charges [45-53]. But 
that's not all. Consecutive consideration of this issue gives arguments in favor of the possibility of changing the dimensionality of space. The string theory, because of its non-local specifics, contains additional symmetries that are not contained in local theories. The T-duality of the string theory imposes a ban on the possibility of an electric field strength to accept infinitely large values. In thermodynamic equilibrium, each degree of freedom of the system, on the average, has the same energy (the law of equidistribution). If, for very large energy densities, this law is also valid, this will lead to the fact that a finite number of degrees of freedom for a finite number of fields will contain only a finite energy density. At an infinite energy density of the Big Bang (BB), a finite number of degrees of freedom at the initial moments of the explosion can not accumulate the released energy. As applied to the initial instant of time in the theory of $\mathrm{BB}$, this leads to an infinite (or very large) dimensionality of the space

$$
N(t) \geq 16 \pi^{3} \alpha^{\prime 2} w(t)
$$

where $\alpha^{\prime}$ is so called slope parameter in string theory, which is proportional to the string tension, and $w(t)$ is an energy density of $\mathrm{BB}$ at some time moment $t$ near BB initiation, and, consequently, to the possibility of changing it $[63,64]$. The change in the dimension of space gives, in turn, the key to describing the nature of the cosmological constant [63, 64]. Thus, the phenomena of micro- and macrocosm are related in a non-trivial way.

Of all the above mentioned set of characteristic manifestations of the non-locality of our world, we will be interested below in two, it seems to us, closely related questions - the question of the nature of the corpuscular-wave duality and the question of the nature of the Heisenberg uncertainty relations.

\subsection{Wave Description of Particles in a Non-Local Approach}

It was noted above that the conclusion that particles should exhibit wave properties was obtained by de-Broglie from the analogy of the description of particles in classical mechanics and the wave motion of light. This analogy is the heuristic factor of such a substantiation of the wave properties of particles. There is no derivation as such in the local approach. The derivation of the wave equation for particles in a non-local approach is contained, for example, in $[52,65]$. We briefly recall the derivation of this equation in the non-local approach of string theory. A string is a line can be specified as $X^{\mu}, \mu=1,2, \ldots$, $N$, maps in the enclosing space $\left(x_{1}, x_{2}, \ldots x_{N-1}, t\right)$. When constructing the density of the Lagrangian of string theory, called the Nambu-Goto Lagrangian density, it is assumed that its most general form is proportional to the area swept out by the string as it moves and has a form

$$
\tilde{L}\left(\dot{X}^{\mu}, X^{\mu \prime}\right)=-\frac{T_{0}}{c} \sqrt{\left(\dot{X} \cdot X^{\prime}\right)^{2}-(\dot{X})^{2}\left(X^{\prime}\right)^{2}}
$$

where

$$
\begin{gathered}
\dot{X}^{\mu}=\frac{\partial X^{\mu}}{\partial \tau} \\
X^{\mu \prime}=\frac{\partial X^{\mu}}{\partial \sigma}
\end{gathered}
$$

$T_{0}$ is the string tension, $c$ is the speed of light, $\tau, \sigma$-variables responsible for string parameterization. The standard view of a relativistic scalar product from [52] is used

$$
(a \cdot b)=a_{\mu} b^{\mu}=a_{0} b^{0}+a_{1} b^{1}+a_{2} b^{2}+a_{3} b^{3}=-a^{0} b^{0}+\vec{a} \vec{b}=-a^{0} b^{0}+a^{1} b^{1}+a^{2} b^{2}+a^{3} b^{3}
$$

Accordingly, the action for describing the string is represented in the form

$$
S=\int_{\tau_{i}}^{\tau_{f}} d \tau \int_{0}^{\sigma_{1}} d \sigma \tilde{L}\left(\dot{X}^{\mu}, X^{\mu \prime}\right)
$$

The equations of motion of a string are obtained from the condition of equality to zero of the action variation

where

$$
\frac{\partial P_{\mu}^{\tau}}{\partial \tau}+\frac{\partial P_{\mu}^{\sigma}}{\partial \sigma}=0
$$




$$
\begin{gathered}
P_{\mu}^{\tau} \equiv \frac{\partial \tilde{L}}{\partial \dot{X}^{\mu}}=-\frac{T_{0}}{c} \frac{\left(\dot{X} \cdot X^{\prime}\right) X_{\mu}^{\prime}-\left(X^{\prime}\right)^{2} \dot{X}_{\mu}}{\sqrt{\left(\dot{X} \cdot X^{\prime}\right)^{2}-(\dot{X})^{2}\left(X^{\prime}\right)^{2}}} \\
P_{\mu}^{\sigma} \equiv \frac{\partial \tilde{L}}{\partial X^{\mu \prime}}=-\frac{T_{0}}{c} \frac{\left(\dot{X} \cdot X^{\prime}\right) \dot{X}_{\mu}-(\dot{X})^{2} X_{\mu}{ }^{\prime}}{\sqrt{\left(\dot{X} \cdot X^{\prime}\right)^{2}-(\dot{X})^{2}\left(X^{\prime}\right)^{2}}}
\end{gathered}
$$

When using action in terms of transverse velocity in static calibration, we obtain

$$
\frac{1}{c^{2}} \frac{\partial^{2} \vec{X}}{\partial t^{2}}=\frac{\sqrt{1-\frac{v_{\perp}^{2}}{c^{2}}}}{\frac{\partial s}{\partial \sigma}} \frac{\partial}{\partial \sigma}\left[\frac{\sqrt{1-\frac{v_{\perp}^{2}}{c^{2}}}}{\frac{\partial s}{\partial \sigma}} \frac{\partial \vec{X}}{\partial \sigma}\right]
$$

where $s$ is a length parameter along a string, $\vec{v}_{\perp}=\frac{\partial \vec{X}}{\partial t}$ is perpendicular to the string for all its points.

Let us define the timeless quantity $A(\sigma)$

$$
A(\sigma)=\frac{\frac{\partial s}{\partial \sigma}}{\sqrt{1-\frac{v_{\perp}^{2}}{c^{2}}}}
$$

The choice of parametrization, in which the parameter is the length of the string, is natural, but in the general case we can change the scale of the motion along the string so that $\sigma \propto s$. We choose $\sigma$ so that $A \equiv c / \tilde{c}$, where $\tilde{c}$ is the speed of a wave described by a string. Then from (1) we have

$$
\frac{1}{\tilde{c}^{2}} \frac{\partial^{2} \vec{X}}{\partial t^{2}}=\frac{\partial^{2} \vec{X}}{\partial \sigma^{2}}
$$

Note that the derivation of the wave equation for $\tilde{c}=c$ is contained, for example, in [52]. A simple generalization to arbitrary speeds is contained in [65]. It can be seen that by choosing $\tilde{c}$ for $A$ we can make the propagation velocity of the wave arbitrary in the resulting wave equation (in [52] the analysis ends with the condition $A=1$ ). On the one hand, it may seem that there is something artificial in this arbitrariness. On the other hand, this can be considered as the circumstance that we can describe by wave a particle moving with arbitrary velocities.

\subsection{Heisenberg Uncertainty Relations}

As a consequence of a probabilistic description, we receive the Heisenberg uncertainty relations [3, 66-74]. There are some disagreements in the interpretation of the relationship on the coordinates-momenta and energy-time. However, despite the difference in their interpretation by some authors, they formally have the same form. The impossibility of an accurate prediction of the characteristics of a particle it is natural to associate with the fact that it does not represent a local object.

Local approach. The justification of the Heisenberg uncertainty relations is contained, for example, in [3] and can be seen from relation

$$
m_{A} m_{B} \geq \frac{1}{2}|<[A, B]>|
$$

in the case of non-commuting operators $A$ and $B$, where $m_{A}, m_{B}$ are the square root of the dispersions of the physical values $A$ and $B$, on the right side there is an average value of the commutator of the operators corresponding to the given values.

The uncertainty relation obtained by Heisenberg had the form

$$
\Delta p \Delta x \geq h
$$

The same relation was obtained in a large number of papers by authors who considered quantum effects in various physical problems [3, 66]. A significant refinement was obtained in $[67,68]$, where for Hermitian conjugate operators $A$ and $B$ took place

$$
m_{A} m_{B} \geq \hbar / 2=h / 4 \pi
$$


For arbitrary operators, there was a relation

$$
\left(m_{A} m_{B}\right)^{2}-\left(m_{A B}\right)^{2} \geq \hbar^{2} / 4
$$

It is known, for example, that equality in the relation (2) for the coordinate and momentum is realized for Gaussian wave packets belonging to pure states. In the relation (3), the equality sign is achieved, generally speaking, also for pure states. A substantial understanding of the difference between mixed and pure states is analyzed in detail in [70-73]. If the degree of "purity" of the state is defined as

$$
\mu=S p \hat{\rho}^{2}
$$

where $\hat{\rho}$ is the statistical operator, $S p \hat{\rho}=1$ (for pure quantum states $\mu=1$ ), then it is possible to obtain the relation

$$
m_{p} m_{x} \geq \frac{4}{9 \mu} \hbar
$$

If we take into account the correlation coefficient, then this ratio is generalized to the form

$$
\sqrt{m_{p}^{2} m_{x}^{2}-m_{p x}^{2}} \geq \frac{4}{9 \mu} \hbar
$$

If the degree of "purity" is defined as

$$
\mu_{q}=\left(S p \widehat{\rho}^{q}\right)^{1 / q}
$$

where $q$ is an arbitrary number, then we can get the inequality

$$
m_{p} m_{x} \mu_{q}^{p} \geq \hbar\left(1+\frac{1}{p}\right)^{-p}
$$

where

$$
\frac{1}{p}+\frac{1}{q}=1
$$

Inequality (4) correspond to this inequality for $p=q=2$. The best inequality is obtained when $\mu=\infty$, since $\mu_{\infty}<\mu_{q}, 1 \leq \mathrm{q}<\infty$, and represents

$$
m_{p} m_{x} \mu_{\infty} \geq \frac{\hbar}{2}
$$

It turns out that (4) is an asymptotic form of a more general inequality

$$
m_{p} m_{x} \geq \frac{\hbar}{2} \Phi(\mu)
$$

when $\mu<<1$. The function $\Phi$ is a monotonously increasing function with decreasing $\mu(0<\mu \leq 1)$, $\Phi(1)=1$. When $\mu<<1$, it has the form

$$
\Phi(\mu)=\frac{8}{9 \mu}\left(1+\frac{9}{64} \mu^{2}+\ldots\right)
$$

It can be seen that the calculation of the coefficient for $\mathrm{h}$ in the Heisenberg inequality is in general a difficult task for any particular system. Therefore, if this coefficient is not principle to the consideration, the form follows from (2)

$$
\Delta p \Delta x \geq \frac{\hbar}{2}
$$

is often used.

However, the Schrödinger equation in the local approach is not derived, but postulated. The matrix mechanics of Heisenberg is equivalent to the Schrödinger approach. Consequently, matrix mechanics is also postulated together with the presence of non-commuting operators in it. Thus, the nature of the uncertainty relations hides behind the nature of the Schrödinger equation.

Nonlocal approach. Earlier [65], we mentioned the close connection between the wave behavior of particles and the Heisenberg uncertainty relations. Note, that in the overwhelming number of attempts to disprove the uncertainty relations, for example, in Bohr's disputes with Einstein (see also the supplement to the Russian edition [66]), thought experiments with light exhibiting wave properties took place. 
This suggests that the wave nature of particles (massless (photons) or massive) is closely related to the nature of the uncertainty relation. If the uncertainty relations are a consequence of non-locality, then the wave structure of particles (both mass and massless) should be also described in a non-local way. Conversely, if the wave nature of the particles can be explained in a non-local description, then, since it must be closely related to the nature of the Heisenberg uncertainty relations, the latter can also be clarified in a non-local description. Indeed, it can be shown that the both wave nature of particles and the nature of uncertainty relations can be explained in a nonlocal approach [65]. In this case (for more details, see [65]) in the space-time $\mathrm{N}$ dimensional space with one-dimensional time we get

$$
|\Delta \vec{p} \Delta \vec{s}|=\alpha\left\langle\sqrt{1-\frac{v_{\perp}^{2}}{c^{2}}}\right\rangle h
$$

where $\Delta p$ and $\Delta s$ are, respectively, the characteristic ranges of the momentum and the coordinates spread of the various sections of the string, angular brackets indicate the mean value of the value in brackets, $\alpha$ is a certain coefficient of the order of unity arising as a result of averaging. Let us decompose the vectors $\Delta p$ and $\Delta s$

$$
\begin{aligned}
\Delta \vec{p} & =\sum_{\mu=1}^{N-1} \Delta p_{\mu} \vec{e}_{\mu} \\
\Delta \vec{s} & =\sum_{\mu=1}^{N-1} \Delta s_{\mu} \vec{e}_{\mu}
\end{aligned}
$$

with respect to some orthonormal system $e_{\mu}$, where $(N-1)$ is the spatial dimension of the space under consideration (of full dimension $N$ ), we obtain

$$
\left|\sum_{\mu=1}^{N-1} \Delta p_{\mu} \Delta s_{\mu}\right|=\alpha\left\langle\sqrt{1-\frac{v_{\perp}^{2}}{c^{2}}}\right\rangle h
$$

If the string is not strongly deformed during its movement, then

$$
\left|\sum_{\mu=1}^{N-1} \Delta p_{\mu} \Delta s_{\mu}\right| \approx\left\langle\sqrt{1-\frac{v_{\perp}^{2}}{c^{2}}}\right\rangle h
$$

If, moreover, it is non-relativistic, then

$$
\left|\sum_{\mu=1}^{N-1} \Delta p_{\mu} \Delta s_{\mu}\right|=h
$$

Additional (compactificated) dimensions should be different from those observed. But what this difference is, we can not yet say, because we do not have any data on the actual structure of the additional dimensions. Therefore, we will assume that none of the spatial components is distinguished with respect to the others. Not distinguished in the sense of its contribution to the presented sum, of course, explicit and implicit dimensions differ in that we observe some of them in everyday experience, while others do not. Then the relation under consideration becomes

accordingly, for energy-time

$$
\Delta p_{\mu} \Delta s_{\mu} \approx \frac{h}{N-1}
$$

$$
\Delta E \Delta t \approx \frac{h}{N-1}
$$

At the moment it is assumed that the dimension of our space $[45-53,63,64]$ is 11 . Then the coefficient on the right-hand side is equal to

$$
\frac{h}{10}
$$

and is in good agreement with the coefficient adopted by far in the overwhelming number of monographs on theoretical physics

$$
\frac{\hbar}{2}=\frac{h}{4 \pi}
$$


In [35] it was noted that a multidimensional space is a kind of bridge connecting the phenomena of the micro- and macroworld. The non-locality of our world leads in nonlocal description to multidimensional spaces, the possibility of changing the dimension - to clarify the nature of the cosmological constant. However, the specific size of the dimension did not appear in this case at all. In considering the nature of the Heisenberg uncertainty relations in the non-local approach, it turns out that the correct coefficient in this relation is obtained only for a certain dimensionality of space, which coincides with the dimension obtained when considering the internal logic of constructing a non-local theory.

\section{Conclusions}

1. At the initial stage, the concept of a particle as a corpuscle led to the formation of a local approach to the description of the physical nature of the world around us and contributed to the advancement of scientific consideration of physical laws. As the understanding of the physical structure progressed, physics encountered another manifestation of particles - the wave.

2. An attempt to describe the joint manifestation of the corpuscle and wave behavior of particles first leads in the local description to the principle of complementarity. Careful analysis of corpuscular-wave duality phenomena together with other phenomena ultimately leads to an understanding of the nonlocality of interactions in the microworld.

3. The stage of consideration of physics, based on the construction of non-local Lagrange functions, begins. The simplest non-local structure is the line; accordingly, this stage is characterized by the development of string theories.

4. String theory cannot exist in a space of arbitrary dimension. Thus, the non-locality of the phenomena of the microworld dictates the consideration of multidimensional spaces, the dimension of which substantially exceeds the dimension of the space surrounding us, perceived by our senses. In this regard, it can be said that the first historical evidence of the multidimensionality of our space is associated with the stage of formation of quantum mechanics. In the local description, we also come to multidimensional spaces, but in this case the transition to multidimensional spaces can be interpreted as the possibility of a more convenient description of the processes under consideration.

5. Additional symmetries of string theories that are not contained in the local consideration, when analyzing the phenomena accompanying the tremendous release of energy of the Big Bang, allow us to conclude that the dimension of space can be changed. Heisenberg, analyzing the achievements of modern physics, noted that the thesis of Heraclitus about fire as primary matter almost exactly corresponds to the statements of modern science, if we replace the word "fire" with the word "energy". Now we can extend Heraclitus-Heisenberg's statement, adding that energy is also the source of space and its dimension.

6. If it is possible to change the dimension of space, the meaning of the cosmological constant is clarified. Cosmological constant from the mathematical point of view can be interpreted as Lagrange's multiplier describing transition of action in space of higher to action in space of lower dimension. From the physical point of view upon transition to space of lower dimension the energy associated with disappearing degrees of freedom has to be extracted in the remained space. This energy is naturally associated with the cosmological constant.

7. In this way, a multidimensional space is a kind of bridge connecting the phenomena of the micro- and macroworld. The non-locality of our world leads in nonlocal description to multidimensional spaces, the possibility of changing the dimension - to clarify the nature of the cosmological constant. Thus, the phenomena of micro- and macrocosm are related in a non-trivial way.

8. Most results in quantum mechanics are obtained in the local approach. The most intriguing of these are the corpuscular-wave duality and the Heisenberg uncertainty relations. However, there is no explanation for them in the local approach. The nature of these physical phenomena is hidden behind the nature of the Schrödinger equation, which is actually postulated.

9. It is natural to look for the explanation of non-local phenomena in a non-local rather than a local approach. The non-local approach leads to a wave equation for the particle. At the beginning of the appearance of the uncertainty relations in Bohr's disputes with Einstein, thought experiments with particles are featured. This suggests that the nature of the particles should be closely related to the 
nature of the uncertainty relations. In other words, if in the non-local approach an explanation is found for the wave behavior of particles, then the same approach should contain an explanation of the uncertainty relations.

10. It turned out that a non-local approach does provide an opportunity to derive uncertainty relations. Explanations both of the wave behavior of particles and the Heisenberg uncertainty relations are based on a non-local approach to the action in the form of Nambu-Goto. Only the area swept by the string during its movement appears in it.

11. In the uncertainty relations obtained in a non-local approach, Planck's constant contains a coefficient that depends on the dimensionality of our space. Modern local and nonlocal theories lead to the dimension of our space equal to 11. Given the equivalence of the contribution of explicit and compactificated dimensions to these relations, this coefficient practically becomes equal to the coefficient used in the vast majority of existing monographs and papers on quantum mechanics.

12. The modern interpretation of corpuscular-wave duality is reflected in the complementarity principle, based on the Heisenberg uncertainty relations. These relations were obtained in the non-local approach of string theory; therefore, together with the derivation of the wave equation in the non-local approach, the main obstacles to the conceptual justification of corpuscular-wave duality have been overcome. Thus, both the corpuscular-wave duality and the Heisenberg uncertainty relations receive their natural explanation in the non-local approach.

13. Thus, at the present stage of research, a particle can be quite consistently represented by a string. It is enough that, on the basis of the non-locality of our world, both the uncertainty relation and the copruscular-wave duality of particle behavior are explained. To what extent the particle contains nonlocal objects of a higher order than the string - a membranes of dimension $n>1$ has yet to be clarified. 14. Thanks to this interpretation of the nature of the particles, a part and the whole universe, macro and microphysics, are combined. A particle as part of the universe reflects the whole universe. As a nonlocal object, it dictates the need for a multi-dimensional space, and a completely specific dimension, i.e. through this multidimensionality it bears on itself the seal of the comprehensive space in which it is located and evolves. Macro and microphysics phenomena are not mutually exclusive, i.e. they are not intersecting regions of this whole, but turn out to be closely interconnected through the multidimensionality of space, which manifests itself in a part of this whole, i.e. in the nature of particles.

\section{References}

1. W. Heisenberg, Physik und Philosophie. Frankfurt am Main, 1959.

2. H. Poincare, Valeur de la science, 1905.

3. L. De Broglie, Les Incertitudes D'Heisenberg Et L'Interpretation Probabiliste De La Macanique Ondulatore. Gauthier-Villars, Bordas, Paris, 1982. (Heisenberg uncertainty relations and probability interpretation of wave mechanics) (in French).

4. N. Bohr, H. Kramers, C. Slater, Philosophical Maqazin, vol. 47, p. 485, 1924.

5. N. Bohr, H. Kramers, C. Slater, Zeits. f. Phys., vol. 24, p. 69, 1924.

6. L. De Broglie, "Will quantum mechanics remain indeterministic?" Report delivered at the Synthesis Center on October 3, 1952. In: Selected scientific works. - vol. 4. Thermodynamics of an isolated particle. Reinterpretation of wave mechanics. Reports and speeches. Moscow: Publishing house "PRINT-ATELIER", 2014. pp. 239-255 (in Russian).

7. V. V. Belokurov, O. D. Timofeevskaya, O. A. Khrustalev, Quantum teleportation is an ordinary miracle. Izhevsk: "Regular and Chaotic Dynamics", 2000 (in Russian).

8. L. De Broglie, "The duality of waves and particles in the works of Einstein." Lecture at the Academy of Sciences at a public meeting on December 5, 1955. In: Selected scientific works. - vol. 4. Thermodynamics of an isolated particle. Reinterpretation of wave mechanics. Reports and speeches. Moscow: Publishing house "PRINT-ATELIER", 2014, pp. 256-276 (in Russian).

9. W. Heizenberg, Die Naturwissenschaften, vol. 17, p. 490, 1929.

10. G. Greenstein, A. G. Zajonc, The quantum challenge. Modern research on the foundations of quantum mechanics. Jones and Barlett Publishers, Inc., 2006.

11. W. E. Jr. Lamb, M. O. Scully, "The photoelectric effect without photons." In: Polarisation, Matiere et Rayonnement. Presses University de France, 1969. 
12. L. Mandel, "The case for and against semiclassical radiation theory." Progress in Optics, vol. 13, Amsterdam: Notth-Holland, 1976.

13. M. D. Crisp, E. T. Janes, "Radiative effects in semiclassical theory." Phys. Rev., vol. 179. pp. 1253-1261, 1969.

14. L. I. Gudzenko, S. I. Yakovlenko, Plasma lasers. Moscow: Atomizdat, 1978, (in Russian).

15. B. F. Gordiets, A. I. Osipov, L. A. Shelepin, Kinetic processes in gases and molecular lasers. Moscow: "Nauka", 1980 (in Russian).

16. S. I. Yakovlenko "Gas and plasma lasers." In: Encyclopedia of low-temperature plasma. / Ed. V.E. Fortov. Introductory volume - vol. IV. M: "Nauka" MAIK " Nauka / Interperiodica", 2000, pp. 262-291 (in Russian).

17. Encyclopedia of low-temperature plasma, Series B: reference applications, databases and databases. Ch. ed. Fortov V.E. Vol. XI-4: Gas and Plasma Lasers. Ed. Yakovlenko S.I. Moscow: "Fizmatlit", 2005 (in Russian).

18. V. M. Batenin, V. V. Buchanov, A. M. Boichenko, M. A. Kazaryan, I. I. Klimovskii, E. I. Molodykh, Highbrightness metal vapour lasers: Physical fundamentals and mathematical models. CISP: CRC Press Taylor \& Francis Group (Boca Raton, London, New York), vol. 1, 2017.

19. A. M. Boichenko, A. N. Panchenko, V. F. Tarasenko, A. N. Tkachev, S. I. Yakovlenko, N. A. Panchenko, Gas and plasma lasers. Monographic series "Radiation. Beams. Plasma.". Issue 2. Tomsk: "STT Publishing", 2017 (in Russian).

20. A. M. Boichenko, M. I. Lomaev, A. N. Panchenko, E. A. Sosnin, V. F. Tarasenko, UV and VUV excilamps: Physics, technique and applications. Tomsk: "STT Publishing", 2011 (in Russian).

21. A. M. Boichenko, Lamp Emission Sources. Theoretical description. LAP LAMBERT, Academic Publishing, 2018 (in Russian).

22. W. Glaser, Grundlagen der elektronenoptik. Wien, Springer-Verlag, 1952.

23. Runaway electron beams and discharges based on the background electron multiplication wave in dense gases. Ed. Yakovlenko S.I., Proceedings of IOFAN, vol. 63, Moscow: "Nauka", 2007 (in Russian).

24. A. M. Boichenko, A. N. Tkachev, S. I. Yakovlenko, V. F. Tarasenko, Ch. 2: "Non-local criterion for electron runaway." In: Generation of Runaway Electrons and X-Rays in the Discharges of High Pressure, Ed. Tarasenko V.F., Tomsk: STT Publishing, 2015, pp. 55-78. (in Russian).

25. A. M. Boichenko, A. N. Tkachev, S. I. Yakovlenko, V. F. Tarasenko, E. Kh. Baksht, Ch.10: "Generation of subnanosecond electron beams in gas-filled and vacuum diodes." In: Generation of Runaway Electrons and Xrays in the Discharges of High Pressure. Ed. Tarasenko V.F., Tomsk: STT Publishing, 2015, pp. 255-296 (in Russian).

26. A. M. Boichenko, A. N. Tkachev, S. I. Yakovlenko, Ch. 2: "Generation of Powerful Runaway Electron Beams in Dense Gases." In: Physics Research and Technology. Runaway Electron Beams and X-rays in High Pressure gases. vol. 2. Processes and Applications. Editor: Tarasenko V.F., Nova Science Publishers, NY, USA, 2016, pp. 41-106.

27. V. P. Vizgin, Unified field theories in a quantum-relativistic revolution. The program of field historical synthesis of physics. Moscow: URSS, 2017 (in Russian).

28. O. N. Krokhin, "On the nature of the photon." Physical education in universities, vol. 21, no. 3, pp. 19-23, 2015 (in Russian).

29. M. Creutz, Quarks, gluons and lattices. Cambridge University Press, 1983.

30. B. Greene, The fabric of the cosmos. Space, time, and the texture of reality. Alfred A. Knopf, New York, 2014.

31. R. P. Feynman, A. R. Hibbs, Quantum mechanics and path integrals. McGray W-Hill Book Company, New York, 1965.

32. A. Einstein, B. Podolsky, N. Rosen, Phys. Rev., vol. 47, p. 777, 1935.

33. E. Scrodinger, Proc. Cambr. Phil. Soc., vol. 31, p. 555, 1935.

34. J. S. Bell, Physics, vol. 1, p. 195, 1964.

35. A. M. Boichenko, "Local/Nonlocal Descriptions in Physics and Dimension of Space." Phys. Astron. Int. J., vol. 1, no. 6, p. 00034, 2017.

36. Y. Kim, R. Yu, S. Kulik, Y. Shih, M. Scully, Phys. Rev. Lett., vol. 84, pp. 1-5, 2000.

37. M. G. Ivanov, How to understand quantum mechanics? Moscow-Izhevsk: RHD, 2015 (in Russian).

38. A. Aspect, P. Grangier, G. Roger, Phys. Rev. Lett., vol. 47, p. 460, 1981.

39. A. Aspect, P. Grangier, G. Roger, Phys. Rev. Lett., vol. 49, p. 91, 1982.

40. A. Aspect, J. Dalibar, G. Roger, Phys. Rev. Lett., vol. 49, p. 1804, 1982.

41. R.P. Feynman, Quantum electrodynamics. A lecture note. W.A. Benjamin, Inc. New York, 1961.

42. N. N. Bogolyubov, D. V. Shirkov, Introduction to the theory of quantified fields. Moscow: "Nauka", 1984 (in Russian). 
43. S. Weinberg, The quantum theory of fields, vol. 1-3. Cambridge University Press, 2000.

44. M. E. Peskin, D. V. Schroeder, An introduction to quantum field theory. Addison-Wesley Publishing Company, 1995.

45. M. B. Green, J. H. Schwarz, E. Witten. Superstring theory, Cambridge University Press, 1987.

46. G. V. Efremov. Problems of nonlocal interaction quantum theory. Moscow: "Nauka", 1985 (in Russian).

47. B. M. Barbashov, V. V. Nesterenko. Model of relative string in hadron physics. Moscow: "Energoatomizdat", 1987 (in Russian).

48. L. Brink, M. Henneaux. Principles of string theory. Plenum Press, 1988.

49. M. Kaku. Introduction to superstrings. Springer-Verlag, 1988.

50. S. V. Ketov. Nonlinear sigma models in quantum field theory and string theory. Moscow: "Nauka", 1992 (in Russian).

51. K. Becker, M. Becker, J. H. Schwarz. String theory and M-theory. A modern introduction. Cambridge University Press, 2007.

52. B. Zwiebach. A First Course in String Theory. Cambridge University Press, 2004.

53. S. V. Ketov. Introduction to quantum theory of strings and superstrings. Moscow, URSS, 2018 (in Russian).

54. P. A. Collins, R. W. Tucker. Nucl. Phys. vol. B112, no. 1, pp. 150-176, 1976.

55. L. Mlodinow. Feynman's Rainbow. A Search for Beauty in Physics and in Life. Livebook Publishing Ltd, 2014.

56. G. Veneziano. Nuovo Cimento, vol. 57A, p. 190, 1968

57. Y. Nambu In.: Proc. Int. Conf. on Symm. and Quark Models. Wayne State Univ., 1969, Gordon and Breach, London, 1970.

58. H. Nielsen. In: $15^{\text {th }}$ Int. Conf. on Hiqh Enerqy Physics, Kiev, 1970.

59. L. Susskind. Phys. Rev., vol. D1, p. 1182, 1970.

60. P. M. Ramond. Phys. Rev., vol. D3, p. 2415, 1971.

61. A. Neveu, J. H. Schwartz. Nucl. Phys., vol. B31, p. 86, 1971; Phys. Rev., vol. D4, p. 1109, 1971.

62. L. Brink, D. Olive, C. Rebbi, J. Scherk. Phys. Lett., vol. B45, p. 379, 1973.

63. A. M. Boichenko. "Dimension of space. Is it constant?" Phys. J., vol. 1, no. 3, pp. 245-254, 2015.

64. A. M. Boichenko. "The cosmological constant as a consequence of the evolution of space." Russian Physics Journal, vol. 59, no. 8, pp. 1171-1180, 2016.

65. A. M. Boichenko "Derivation of Heisenberg uncertainty relations in the non-local approach of string theory." Asian J. Applied Sci., vol. 11, no. 3, pp. 151-162, 2018.

66. W. Heisenberg. Physical principles of quantum theory. Moscow-Izhevsk: "Regular and chaotic dynamics", 2002 (in Russian).

67. E. Schrödinger. "Zon Heisenbergschen Unscharfeprinzip." Ber. Kgl. Akad. Wiss. Berlin, pp. 296-303, 1930.

68. H. P. Robertson. "A general formulation of the uncertainty principle and its classical interpretation." Phys. Rev. $A$, vol. 35 , no. 5, pp. 667, 1930.

69. A. E. Haas. Materiewellen und Quantenmechanik. Leipzig: Akademishe Verlagsgessellschaft, 1928.

70. V. V. Dodonov, V. I. Man'ko. "Generalizations of uncertainty relations in quantum mechanics." In: Invariants and evolution of nonstationary quantum systems. Ed. in Chief Basov N. G., Ed. Markov M. A. Proceedings FIAN, vol. 183, Moscow: "Nauka", 1987, pp. 5-70 (in Russian).

71. M. J. Bastiaans. "Uncertainty principle for partially coherent light." J. Opt. Soc. Amer., vol. 73, no. 3, pp. 251255,1983

72.M. J. Bastiaans. "Lower bound in the uncertainty principle for partially coherent light." J. Opt. Soc. Amer., vol. 73, no. 10, pp. 1320-1324, 1983.

73. M. J. Bastiaans. "New class of uncertainty relations for partially coherent light." J. Opt. Soc. Amer. A, vol. 1, no. 7, pp. 711-715, 1984.

74. A. M. Boichenko. "Entropy as invariant of dynamic system." Quantum Computers and Computing, vol. 5, no. 1, pp. 65-73, 2005. 